\title{
Color Spaces for Transform-based Image Retrieval
}

\author{
Sanjay N Talbar \\ SSGGS Institute of Engineering \&Technology, \\ Vishnupuri, Nanded 431606, \\ Maharashtra, India
}

\author{
Satishkumar L. Varma \\ Don Bosco Institute of Technolog \\ Kurla, Mumbai 400070 \\ Maharashtra, India
}

\begin{abstract}
The discrete image transforms are used for energy compaction primarily and so used in image data compression. The level of energy in the image depends on level of colors used. In this paper we use two discrete image transforms namely Discrete Hadamard Transform (DHT) and Discrete Wavelet Transform (DWT). These transforms are applied on two different color models namely $\mathrm{HSV}$ and $\mathrm{YCbCr}$ separately in a given large standard database with 1000 images formed from 10 different classes taken from the Corel collection. The proposed features are effective and useful for image indexing and retrieval.
\end{abstract}

\section{General Terms}

Image Indexing and Retrieval, Information Retrieval, Multimedia Retrieval

\section{Keywords}

HSV Color Model, YCbCr Color Model, Discrete Hadamard Transform, Image Indexing, Image Retrieval..

\section{INTRODUCTION}

As more and more digital images are acquired into the internet worked multimedia-computing environment, searching and retrieving of image data based on their information content are essential prior to the development and utilization of an effective image database. So the problem of storage and retrieval needs comparatively more attention. Various systems such as iMATCH [1], IRMOMENT [2] are available today for content based image retrieval (CBIR).

The image database was represented using a set of image attribute, such as color [3] [4], shape [4] [5] [6], texture [7] and layout [8] also. Image indexing using compressed transforms was dealt by J. Berens, G. D. Finlayson and G. Qiu [9]. It uses the standard transform encoding methods (the Karhunen-Loeve transform, the discrete cosine transform [10] [11]. The wavelet transform is treated in [12].

In this paper, two discrete images transform namely DHT and DWT are applied on $\mathrm{HSV}$ and $\mathrm{YCbCr}$. The image indexing and retrieval system is overviewed in section 2. In section 3, we show the retrieval performances. Finally, the summery and future work is given in section 4 .

\section{INDEXING AND RETRIEVAL SYTEM}

The system architecture is given in Fig. 1. The $\mathrm{YCbCr}$ and $\mathrm{HSV}$ components separately are divided into block sizes of $8 * 8$. The DWT and DHT are applied on each of these blocks. The top $2 \times 2$ coefficients form the feature vector. The chi-square [5] is used for distance measure and image retrieval.

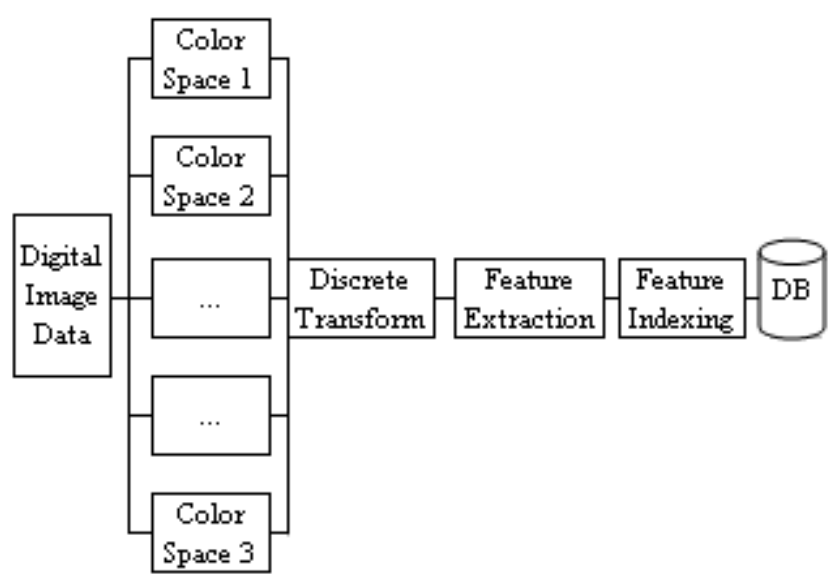

Fig. 1 Image Indexing and Retrieval System Architecture

\subsection{Image Indexing}

The images are then divided into $8^{*} 8$ blocks. The image blocks are then represented from $\mathrm{RGB}$ to the $\mathrm{Y} \mathrm{Cb} \mathrm{Cr}$ format. As images are in JPEG format, we use the DCT and Hadamard domain to develop and extract feature vector for each block of size $8 * 8$ in both the transform domain.

The first four horizionatal elements are used to index the given image for performance comparision and image retrieval system evaluaion. Other directions are also tested. The first four horizonatl elements give better performance in both the cases of DCT and DHT.

\subsection{Image Retrieval}

The Euclidian distance measure [11] between features of query object and database object is used to match and display images. To precision and recall can be used to compare the system performance. It is given in Equation 1 and Equation 2 respectively.

$$
\text { Precision }=\frac{\text { Number of relevant doc umentsretrieved }}{\text { Total number of documentsretrieved }}
$$

$$
\text { Recall }=\frac{\text { Number of relevant documentsretrieved }}{\text { Number of relevant documentsin database }}
$$


To provide numerical results, we tested 30 sample images chosen randomly from ten categories, each containing three of the images. A retrieved image is considered a match if it belongs to the same category of the query image. The categories of images tested are listed in Table 1. Most categories simply include images containing the specified objects.

\section{EXPERIMENTAL RESULTS}

The 100 images each of size $256 * 384$ and $384 * 256$ are taken from 10 semantic groups: tribesmen, elephants, horses, flowers, foods, greek architecture, buses, dinosaurs, snow clad mountains, and beaches. The system results of retrieved images of Dinosaur and Elephant are shown in Fig. 2. The average precision comparison based on the three sample images using feature vectors are plotted in Fig. 3. The average precision using DWT is 0.63 and it is 0.59 in case of DHT. So the DWT performs better than DHT.

\subsection{Database Used}

The proposed system implemented on a database of 1000 images taken from the Corel collection [1][2]. These images were arranged in 10 semantic groups: tribesmen, elephants, horses, flowers, foods, greek architecture, buses, dinoraurs, snow clad mountains, and beaches.

Table 1. Image Class ID and Category

\begin{tabular}{|c|c|}
\hline Group ID & Image Category \\
\hline 1 & Tribal \\
\hline 2 & Beaches \\
\hline 3 & Monuments \\
\hline 4 & Bus \\
\hline 5 & Dinosaur \\
\hline 6 & Elephant \\
\hline 7 & Flower \\
\hline 8 & Horse \\
\hline 9 & Mountains \\
\hline 10 & Food \\
\hline
\end{tabular}

The image database includes around 100 images from each semantic group. The group id for different image category is shown in Table 1 . The images are in JPEG format with two sizes $256 * 384$ and $384 * 256$.

\subsection{Retrieval Using Transforms}

A query image is provided by the user. Query and database images differ in viewpoint, variations in the illumination are present, but rare. The retrieval images using discrete wavelet transform and discrete hadamard transformis are shown in Fig. 2.

\subsection{Performance Comparison}

The 100 images each of size $256 * 384$ and $384 * 256$ are taken from 10 semantic groups: tribesmen, elephants, horses, flowers, foods, greek architecture, buses, dinosaurs, snow clad mountains, and beaches. The average precision comparison based on the three sample images using feature vectors are plotted in Fig. 3. The average precision using DWT is 0.63 and it is 0.59 in case of DHT. So the DWT performs better than DHT.

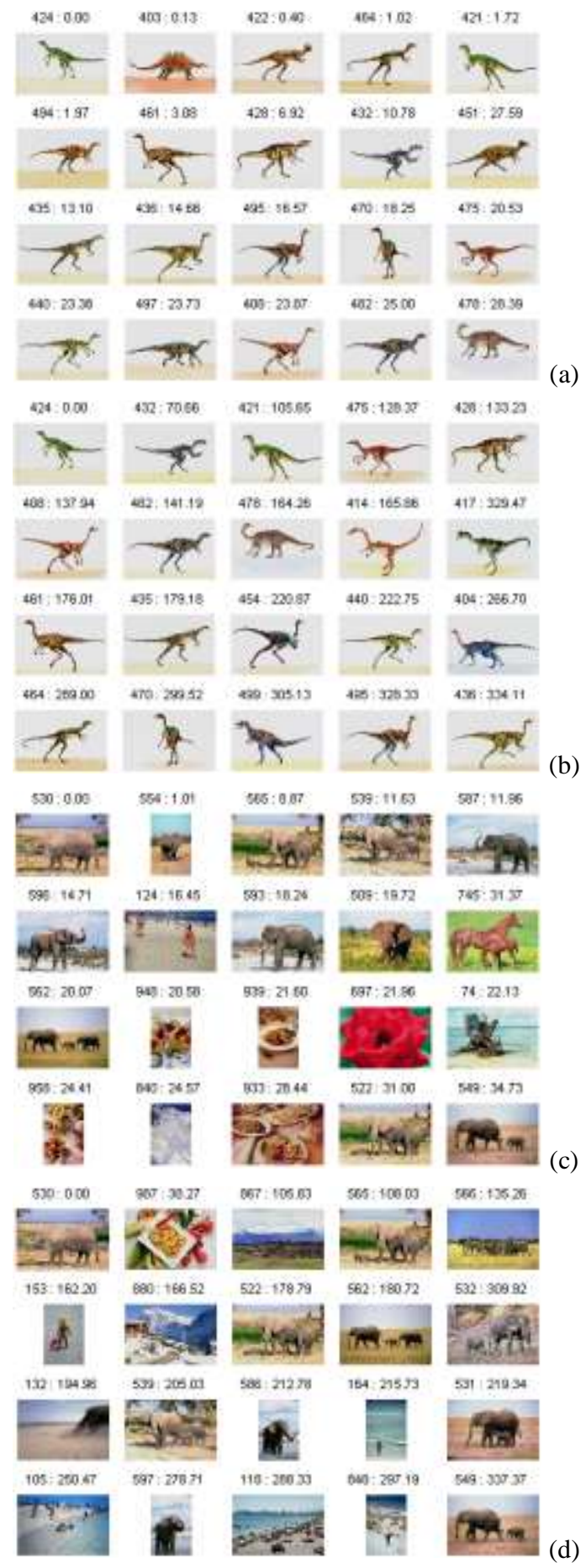

Fig. 2 Retrieval of Dinosaur ( $a$ and b) and Elephant (c and d) as query image using DWT (a and c) and DHT (b and d) 
The estimated precision-recall curve is shown in Fig. 4. The system results of retrieved images using DHT and DWT using Receiver Operator Characteristic (ROC) curve is shown in Fig. 5

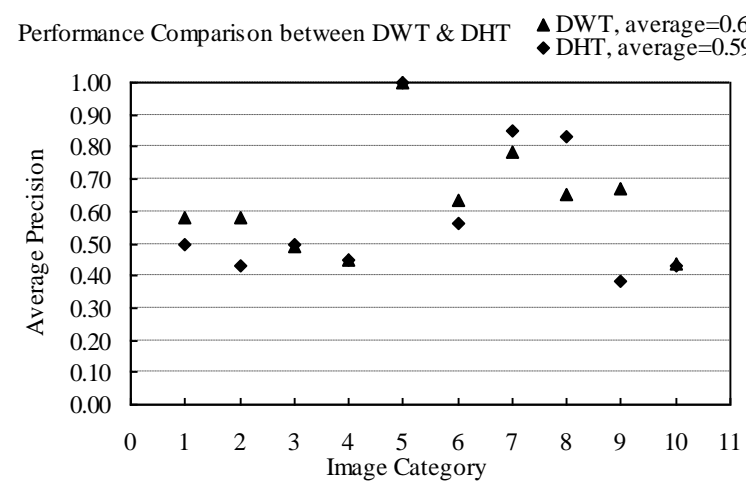

Fig. 3 Performance Comparison between DWT and DHT

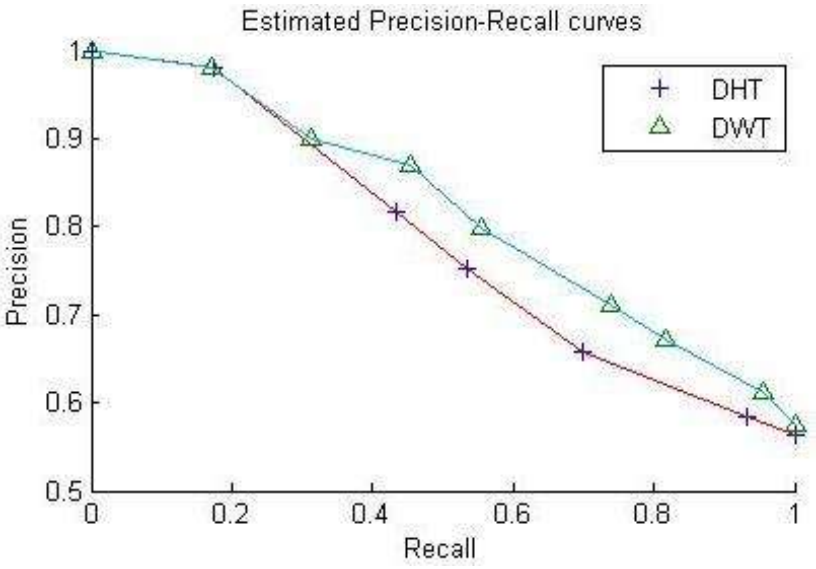

Fig. 4 Estimated Precision-Recall curve

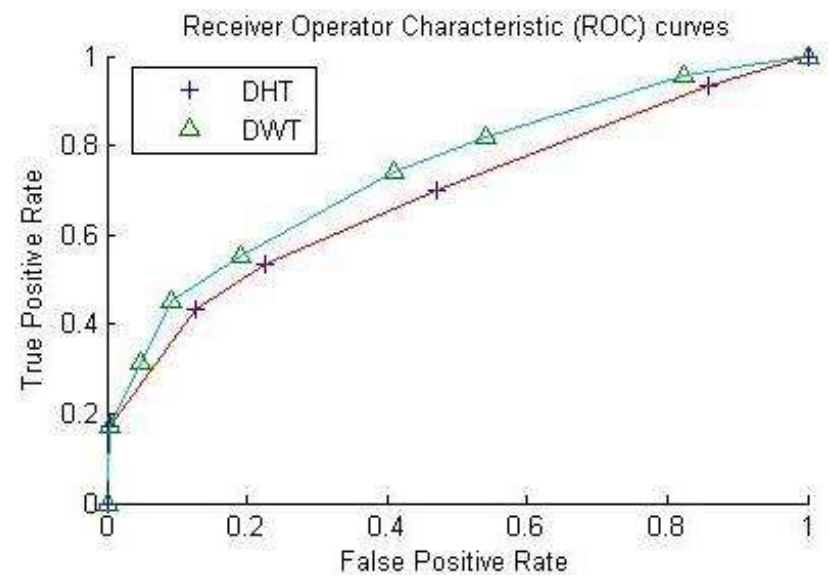

Fig. 5 Estimated ROC curve

\section{CONCLUSION AND FUTURE WORK}

In conclusion, among the many open research challenges and opportunities, one of the most interesting roads ahead for the video retrieval community drives to the Internet. To reach this destination we need to use freely available images and videos labeled. It is observed that the DWT performs better than DHT. Retrieval performance of $100 \%$ is achieved in one of the image category. The results can be further improved by deploying hybrid transforms and using hybrid colors. Combination of different wavelets can also be deployed to improve the results.

\section{ACKNOWLEDGMENTS}

We would like to thank one and all who have helped us to work and develop system for image indexing and retrieval.

\section{REFERENCES}

[1] Sanjay N. Talbar and Satishkumar L. Varma, "iMATCH: Image Matching and Retrieval for Digital Image Libraries," ICETET, pp.196-201, 2009, ISBN: 978-0-7695-3884-6.

[2] Sanjay N. Talbar and Satishkumar L. Varma, "IRMOMENT: image indexing and retrieval by combining moments," IET Digest 2009, 38, DOI:10.1049/ic.2009.0148.

[3] Hossein Nezamabadi-pour and Saeid Saryazdi, "ObjectBased Image Indexing and Retrieval in DCT Domain using Clustering Techniques", Vol. 3 JANUARY 2005 ISSN 1307-6884.

[4] M. J. Swain and D. H. Ballard, "Color indexing", International Journal of Computer Vision, 1991, vol.7, no.1, pp.11-32.

[5] A. K. Jain and A. Vailaya, "Image retrieval using color and shape", Pattern Recognition, 996, vol.29, no.8, pp.12331244.

[6] F. Mokhtarian and S. Abbasi, "Shape similarity retrieval under affine transforms", Pattern Recognition, 2002, vol. 35, pp. 31-41.

[7] B. S. Manjunath and W. Y. Ma, "Texture feature for browsing and retrieval of image data", IEEE PAMI, 1996, no. 18 , vol. 8, pp. 837-842.

[8] J. R. Smith and C. S. Li, "Image classification and querying using composite region templates", Academic Press, Computer Vision and Understanding, 1999, vol.75, pp.165174.

[9] J. Berens, G. D. Finlayson and G. Qiu, "Image indexing using compressed color Histograms", IEEE Proc.-Vision Image Signal Process. Vol. 147, No. 4, August 2000.

[10] Jose A. Lay and Ling Guan, "Image Retrieval Based on Energy Histograms of the Low Frequency DCT Coefficients," IEEE 0-7803-5041-3/99, 1999.

[11] Stepan Obdrzalek and Jiri Matas, "Image Retrieval Using Local Compact DCT-based Representation." DAGM'03, 25th Pattern Recognition Symposium, September 10-12, 2003.

[12] J. Z. Wang, G. Wiederhold, O. Firschein, and X.W. Sha, "Content-Based Image Indexing and Searching Using Daubechies' Wavelets," Int'l J. Digital Libraries, vol. 1, no. 4, pp. 311-328, 1998. 